\title{
Segmentation-Driven 2D-3D Registration for Abdominal Catheter Interventions
}

\author{
Martin Groher ${ }^{1}$, Frederik Bender ${ }^{1}$, Ralf-Thorsten Hoffmann ${ }^{2}$, and Nassir Navab ${ }^{1}$ \\ ${ }^{1}$ Computer Aided Medical Procedures (CAMP), TUM, Munich, Germany \\ $\{$ groher, benderf, navab\}@ics. tum. edu \\ ${ }^{2}$ Department of Clinical Radiology, University-Hospitals Munich-Grosshadern, Germany \\ rthoffma@med.uni-muenchen.de
}

\begin{abstract}
D-3D registration of abdominal angiographic data is a difficult problem due to hard time constraints during the intervention, different vessel contrast in volume and image, and motion blur caused by breathing. We propose a novel method for aligning 2D Digitally Subtracted Angiograms (DSA) to Computed Tomography Angiography (CTA) volumes, which requires no user interaction intrainterventionally. In an iterative process, we link 2D segmentation and 2D-3D registration using a probability map, which creates a common feature space where outliers in 2D and 3D are discarded consequently. Unlike other approaches, we keep user interaction low while high capture range and robustness against vessel variability and deformation are maintained. Tests on five patient data sets and a comparison to two recently proposed methods show the good performance of our method.
\end{abstract}

\section{Introduction}

Catheter-guided interventions are carried out on an every-day basis in almost every hospital throughout the world. Common practice in these interventions is to acquire 2D fluoroscopic sequences of the patient in which catheter, contrasted vessels, and patient anatomy can be visualized for navigation. Also, in order to provide a high resolution visualization of the vasculature only, DSAs are taken. A 3D scan (CTA) of the patient is usually acquired preoperatively in order to evaluate possible risks and plan the treatment. In abdominal catheterizations (e.g. Transarterial Chemoembolization (TACE), or Transjugular Portosystemic Shunt (TIPS)) mono-plane X-ray imaging devices are used more often in contrast to neuroradiology interventions where biplane systems are commonly utilized. Only guided by 2D projections of one view, it is often very difficult for the physician to find a path through the patient's vessel system to the region of interest. This is mainly due to overlay of vessel structures and, in the case of abdominal procedures, the breathing deformation of vessel systems. In order to provide a catheter guidance in 3D, or the transfer of planned information to intraoperative 2D projections, a $2 \mathrm{D}-3 \mathrm{D}$ registration is needed to align $3 \mathrm{D}$ preoperative to $2 \mathrm{D}$ intraoperative data set.

Problems and challenges for this data fusion are as follows. First, due to hard time constraints during the intervention, a 2D-3D registration algorithm should be fast and as automatic as possible during the treatment. Second, vessel features, which are the only features that can be used for DSA-to-CTA registration, are rather distinct in the two

N. Ayache, S. Ourselin, A. Maeder (Eds.): MICCAI 2007, Part II, LNCS 4792, pp. 527 535, 2007.

(c) Springer-Verlag Berlin Heidelberg 2007 
data sets because contrast is injected globally in the preoperative, and locally (through the catheter) in the intraoperative case. A naive registration would not be able to deal with these outliers emerging in $2 \mathrm{D}$ as well as in $3 \mathrm{D}$ and could drive the registration to "false positives". Third, an automatic extraction of vessel features in the 2D DSA would also segment other tubular structures (needles, catheters), which would disturb a feature-based registration. Fourth, due to patient breathing, structures are deformed in the $2 \mathrm{D}$ data set, when compared to the $3 \mathrm{D}$ data set. $2 \mathrm{D}-3 \mathrm{D}$ rigid registration in deformable regions can be addressed by a fully intensity-based procedure with gating, as proposed e.g. by Turgeon et al. [1] for heart. However, it is difficult to use such an image-based method without gating information. In fact, the more appropriate approach to follow would be (partly) feature-based to be able to be robust against deformations. Recently, two methods for 2D-3D registration in the particular case of abdominal angiographic alignment were proposed by Jomier et al. [2] and Groher et al. [3]. The first is a model-to-image technique, which only requires a preoperative segmentation step for aligning DSA images of two views. The second is feature-based aligning extracted 2D and 3D vessel graphs in a one-view scenario. The former is fully automatic during the intervention but suffers from a small capture range while the latter has high capture range but requires manual interaction intraoperatively. As will be shown in section 3 both methods have difficulties to deal with outliers in 3D.

Contribution: We propose a method for 2D-3D registration of preoperative CTA to intraoperative DSA images for abdominal interventions. It automatically aligns a preoperatively segmented 3D vasculature to a 2D DSA image by iteratively segmenting the image and aligning the extracted $2 \mathrm{D}$ and $3 \mathrm{D}$ vasculatures. A combination of registration and 2D segmentation via a probability map allows us to adjust the feature spaces such that non-corresponding features in 2D as well as 3D vasculature are removed consequently. With this approach we combine robustness and high capture range with a fully automatic registration technique. Moreover, one-to-one correspondence of vascular features is assured, which makes it possible to visualize roadmaps in 3D. We motivate our method through an maximum likelihood (ML) formulation solving for both registration and segmentation. Unlike traditional ML-based algorithms for combined segmentation/registration, we only care about the resulting registration and also leave the algorithm as generic as possible in order to use alternative registration and segmentation steps.

Related Work: A combination of segmentation and 2D-3D registration was proposed by Hamadeh et al. [4] and Bansal et al. [5] for the rigid alignment of medical images. The former only segmented once for aiding the registration, the latter used a minimax-approach in order to optimize one energy functional that encapsulates the joint conditional entropy of DRR and X-ray given a segmentation. A recent method proposed by Brox et al. [6] combines pose estimation with a level set formulation to let a registration aid the segmentation. In all these methods, the segmentation is integrated into the algorithm and cannot be replaced. Since vessel segmentation is a specific problem where general approaches cannot be applied without modification we tried to leave the combination as generic as possible and discarded these methods. Combined segmentation and registration has also been successfully applied to brain MR images 
(see Pohl et al. [7] and references therein) where an Expectation Maximization (EM) formulation was favored. In contrast to our proposed algorithm, this work also solves for MR specific nuisance parameters and serves a diagnostic application not subject to hard time constraints.

\section{Method}

We now describe our segmentation-driven 2D-3D registration algorithm. We first justify our approach via an ML formulation integrating a segmentation into the registration process and derive a generic algorithm. In section 2.2 we apply our algorithm to 2D-3D DSA-to-CTA registration for abdominal interventions.

\subsection{MLE with Labelmaps}

We want to maximize the probability such that certain registration parameters $\Theta$ fit best the $2 \mathrm{D}$ image data $I$ and the $3 \mathrm{D}$ model $\mathcal{M}$ :

$$
\hat{\Theta}=\arg \max _{\Theta} P(\Theta \mid I, \mathcal{M})
$$

Maximizing the likelihood of (1), i.e. $\arg \max _{\Theta} P(I, \mathcal{M} \mid \Theta)$, is very difficult if there is no correspondence information between image pixels and model points. Thus, we let a $2 \mathrm{D}$ segmentation aid the estimation. We introduce a random variable $\mathcal{L}$ representing a labelmap over the image $I$. Marginalizing over $\mathcal{L}$ we get

$$
\begin{aligned}
\hat{\Theta} & =\arg \max _{\Theta} \sum_{\mathcal{L}} P(\Theta, \mathcal{L} \mid I, \mathcal{M}) \\
& =\arg \max _{\Theta} \sum_{\mathcal{L}} P(\mathcal{L} \mid I, \mathcal{M}) P(\Theta \mid \mathcal{L}, I, \mathcal{M})
\end{aligned}
$$

using the product rule. From this formulation we can deduce an iterative scheme. If we had values for variable $\mathcal{L}$ given, we could solve the ML of eq. (3). Since $\mathcal{L}$ has to be estimated also, we iterate between expectation estimation (E-step) of the unknown random variable $\mathcal{L}$ and optimization of a cost function (M-step) given this expectation:

$$
\begin{aligned}
& \mathcal{L}^{(t)} \leftarrow \mathbb{E}\left(\mathcal{L} \mid \Theta^{(t-1)}, I, \mathcal{M}\right)=\sum_{\mathcal{L}} \mathcal{L} P\left(\mathcal{L} \mid \Theta^{(t-1)}, I, \mathcal{M}\right) \\
& \Theta^{(t)} \leftarrow \arg \max _{\Theta} P\left(\Theta \mid \mathcal{L}^{(t)}, I, \mathcal{M}\right) \stackrel{M L}{\propto} \arg \max _{\Theta} P\left(\mathcal{L}^{(t)}, I, \mathcal{M} \mid \Theta\right)
\end{aligned}
$$

The M-step (eq. (5)) is rather easy to accomplish since we already have a model in 3D $(\mathcal{M})$ and can determine the MLE using $\mathcal{L}^{(t)}$ in a model-to-model registration. For the Estep (eq. (4)), however, we must determine the expectation value of the labelmap given the last registration and the data. Since this is not straight-forward, we will discuss it in more detail. Assuming spatial independence of pixels in image $I$ (which is common in this context, see [7[5]), we can determine the expectation for each pixel $\mathbf{x}$ separately. If we restrict our segmentation on one object only, we can deduce an indicator variable $\ell_{\mathbf{x}}$ for each pixel $\mathbf{x}$, where 


$$
\ell_{\mathbf{x}}= \begin{cases}1, & \text { if } \mathbf{x} \text { is inside the object } \\ 0, & \text { otherwise }\end{cases}
$$

Thus, the expectation for the label $\ell_{\mathbf{x}}$ of a pixel $\mathbf{x}$ becomes

$$
\begin{aligned}
\mathbb{E}\left(\ell_{\mathbf{x}} \mid \Theta^{(t-1)}, I, \mathcal{M}\right) & =P\left(\ell_{\mathbf{x}}=1 \mid \Theta^{(t-1)}, I, \mathcal{M}\right) \\
& =\alpha P\left(\Theta^{(t-1)} \mid \ell_{\mathbf{x}}=1, I, \mathcal{M}\right) P\left(\ell_{\mathbf{x}}=1 \mid I\right)
\end{aligned}
$$

using Bayes' rule, where $\alpha=P\left(\Theta^{(t-1)} \mid I, \mathcal{M}\right)$, and $\mathcal{M}$ is dropped in the last term of 8 since the segmentation of $I$ is independent of the model. With eq. (8) we can assign the expectation of the segmentation to each pixel and thus get a probability map $I_{\mathcal{L}^{(t)}}$ for $\mathcal{L}^{(t)}$. We can interpret this map as the probability for each pixel to be registered (has a correspondence) to the model, given that it is part of the segmented object combined with the a-priori probability to be part of the segmented object.

Note that we see the expectation as a probability where we joined the registration parameters from the last iteration and the a-priori knowledge of a pixel belonging to an object. We still keep the freedom to choose any kind of binarization technique, which we apply to the probability map. We can give a generic algorithm for the segmentationdriven 2D-3D registration, which we will henceforth refer to as EBM algorithm:

Algorithm EBM: Given an image I, a model $\mathcal{M}$, and initial estimates for the parameters $\Theta^{(0)}$, and labelmap $\mathcal{L}^{(0)}$

Initialize values: $\Theta^{(t-1)} \leftarrow \Theta^{(0)} ; \mathcal{L}^{(t-1)} \leftarrow \mathcal{L}^{(0)}$

Repeat until convergence

E-step: For each pixel $\mathbf{x}$ : if $\ell_{\mathbf{x}}=1$ determine the probability for the

new label using eq. (8), else set probability to zero

B-step: Binarize $I_{\mathcal{L}^{(t)}}$ to get $\mathcal{L}^{(t)}$.

M-step: Register $\mathcal{M}$ to $\mathcal{L}^{(t)}$ starting from $\Theta^{(t-1)}$ to get $\Theta^{(t)}$

Note that our method does not follow the strict formulation of the EM algorithm [8],

$$
\Theta^{(t)}=\arg \max _{\Theta} \sum_{\mathcal{L}} P\left(\mathcal{L} \mid \Theta^{(t-1)}, I, \mathcal{M}\right) P(\mathcal{L}, I, \mathcal{M} \mid \Theta) .
$$

In our algorithm, we directly calculate the expectation of the hidden variable $\mathcal{L}$, whereas EM calculates the expectation of the probability of the complete data $(\mathcal{L}, I, \mathcal{M})$ given the incomplete data $(I, \mathcal{M})$ and an estimate of $\Theta$. Unlike EM, convergence is not proven for our approach. In our experiments, however, the algorithm always converged given suitable termination criteria.

\subsection{Segmentation-Driven 2D-3D Registration on Angiographic Data}

We now describe the single steps of EBM in the particular case of 2D-3D registration of 3D CTA and 2D DSA data sets as illustrated in Fig. 1, The object to be segmented in order to aid the 2D-3D registration is, naturally, the vessels of the DSA. Given a DSA image $I$ (Fig. 1(a) and the vasculature model $\mathcal{M}$ (laid over the DSA in Fig. 1(f) of a CTA volume. The vessel model $\mathcal{M}$ is generated by a region growing step and a centerline extraction. Seed points and thresholds are determined manually. Note that the CTA data set is acquired preoperatively and thus, the extraction of vasculature is 


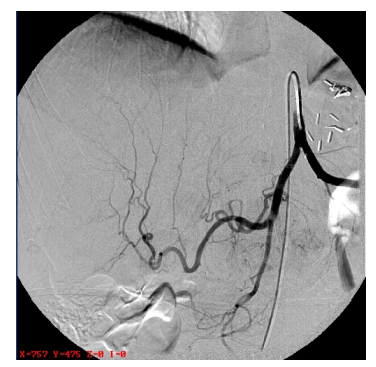

(a)

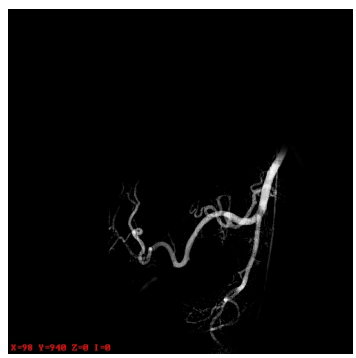

(d)

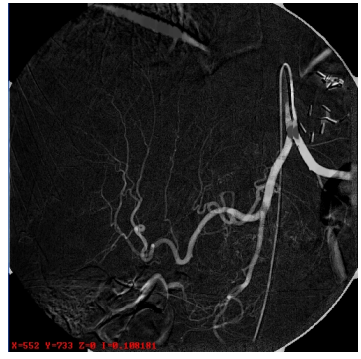

(b)

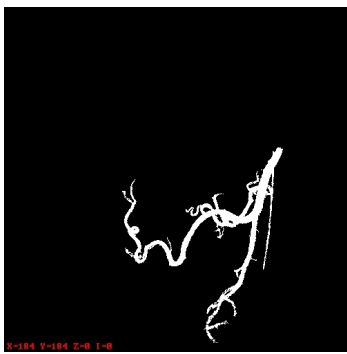

(e)

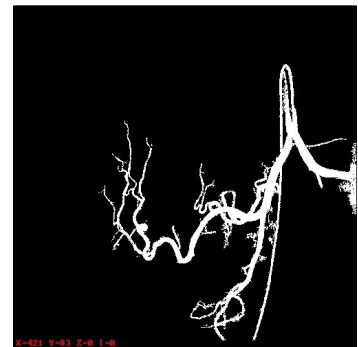

(c)

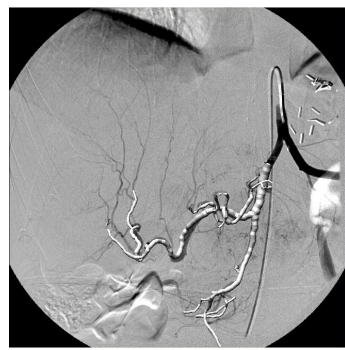

(f)

Fig. 1. Illustration of the segmentation-driven registration:1(a) original DSA, 1(b) bothat filtered DSA, 1(c) initial segmentation (automatic seed point detection, region growing), [1(d) probability map penalizing non-corresponding but extracted features in $2 \mathrm{D}$ and $3 \mathrm{D}, 1(\mathrm{e})$ final segmentation after registration - increased feature similarity, 1(f) overlay of 3D vasculature and DSA

not subject to hard time constraints 1 The $3 \mathrm{D}$ point cloud that spatially describes $\mathcal{M}$, i.e. sampling points on vessel centerlines and bifurcation locations, is denoted by $\left\{\mathbf{X}_{j}\right\}$. We now want to find the rigid registration parameters $\Theta=\left\{\alpha, \beta, \gamma, t_{x}, t_{y}, t_{z}\right\}$ describing the transformation of the model coordinate system into the coordinate system of the C-arm 2 with which the DSA has been acquired. The intrinsic parameters are given and we assume an absence of image distortion due to flat-panel detectors. We consider all image intensities normalized to belong to the domain $\Omega=[0 ; 1]$.

Image Preprocessing: As initialization for the a-priori probability $P\left(\ell_{\mathbf{x}}=1 \mid I_{\mathbf{x}}\right)$ we choose a bothat filtered image whose contours are sharpened by histogram equalization (Fig. 1(b). We refer to this filtered image as $I^{B H}$.

2D Segmentation \& Model Creation: The initial $\left(\mathcal{L}^{(0)}\right.$, see Fig. $\left.1(\mathrm{c})\right]$ as well as all subsequent segmentations of the DSA are computed using a region growing technique based on intensity thresholds. The seed points are detected using a derivative-mean fil-

${ }^{1}$ In fact, the procedure takes about 5 min at most.

${ }^{2}$ Siemens Axiom Artis dFA. 
ter defined by Can et al. [9] for vessel tracing. It detects pixels that are likely to lie on a vessel centerline by filter inspection in 12 directions and criteria evaluation. This method is very fast and yields decent candidate seeds. In order to start with a segmentation, we choose the intensity values for the region growing to be inside the interval $\mu_{\text {seeds }} \pm 2 \sigma_{\text {seeds }}$, which are mean and standard deviation of the intensity values of all detected seed points. We start the region growing from all detected seed points. Outliers are removed by choosing the largest connected region as the actual segmentation. From the segmentation we create a model of a $2 \mathrm{D}$ vessel centerline to be able to register it with the $3 \mathrm{D}$ model $\mathcal{M}$ and deduce a diameter, which is used as $\sigma$ in the E-step. This is done with the same technique as in 3D.

Initial Registration: $\Theta^{(0)}$ is determined by combining information from the C-arm with an exhaustive feature search following the approach proposed by Groher et al. [3].

Iteration: We define the pixel error of a pixel $\mathbf{x}$ as

$$
\varepsilon(\mathbf{x})=d\left(\mathbf{x}, C\left(\mathbf{x},\left\{\mathbf{P}_{\Theta} \mathbf{X}_{\mathbf{j}}\right\}\right)\right)^{2}
$$

where $d(.,$.$) determines the Euclidean distance of two vectors, C\left(\mathbf{y},\left\{\mathbf{z}_{j}\right\}\right)$ determines the closest point of a point set $\left\{\mathbf{z}_{j}\right\}$ to a point $\mathbf{y},\left\{\mathbf{X}_{j}\right\}$ are all points on the $3 \mathrm{D}$ centerline, and $\mathbf{P}_{\Theta}=\mathbf{K}\left[\mathbf{R}_{\Theta} \mid \mathbf{t}_{\Theta}\right]$ is the projection matrix with the current pose parameters $\Theta$.

E-step: This step computes the probability map as defined in eq. (8). The probability that a vessel pixel is registered to the $3 \mathrm{D}$ model, $P\left(\Theta^{(t-1)} \mid \ell_{\mathbf{x}}=1, I, \mathcal{M}\right)$, is defined via the pixel error (eq. (10)). If we allow a deviation proportional to the maximal width of a vessel in the $2 \mathrm{D}$ image, $\sigma$, and assume the error distribution to be Gaussian, we get

$$
P\left(\Theta^{(t-1)} \mid \ell_{\mathbf{x}}=1, I, \mathcal{M}\right)=\frac{1}{\sigma \sqrt{2 \pi}} e^{-\varepsilon(\mathbf{x}) / \sigma^{2}}
$$

The a-priori probability for a pixel $\mathbf{x}$ to lie inside a vessel is defined by the image $I^{B H}$ as described above, i.e. $P\left(\ell_{\mathbf{x}}=1 \mid I\right)=I^{B H}(\mathbf{x})$. Putting both terms together, we define the probability map as

$$
\mathbb{E}\left(\ell_{\mathbf{x}} \mid \Theta^{(t-1)}, I, \mathcal{M}\right) \propto e^{-\varepsilon(\mathbf{x}) / \sigma^{2}} \cdot I^{B H}(\mathbf{x}),
$$

where we dropped $\alpha$ from eq. (8) since it just represents an isotropic scaling in pixel intensities of $I_{\mathcal{L}^{(t)}}$.

B-step: After building up the map (Fig. 1(d)), we perform a new region growing (Fig. 1(e)] and centerline extraction as described above to get a new 2D centerline.

M-step: The 2D-3D registration is computed by minimizing the pixel error $\varepsilon$, which is evaluated only on the $2 \mathrm{D}$ centerline points. If a projected centerline point $\mathbf{P}_{\Theta} \mathbf{X}_{\mathbf{j}}$ already has a closest point, the one with the smaller error is chosen. Thus, we assure one-to-one correspondence of centerline features. The iteration of the registration is governed by a Downhill Simplex optimizer minimizing the non-linear cost function $\sum_{\mathbf{x}} \varepsilon(\mathbf{x})$, where $\mathbf{x}$ has a corresponding (closest) point in the 3D model $\mathcal{M}$.

We stop the algorithm if the absolute difference of the two labelmaps of current and last E-step, $\left|\mathcal{L}^{(t-1)}-\mathcal{L}^{(t)}\right|$, is very small. We choose a threshold of $5 \%$ of the pixels in an image (size $1024^{2}$ ), at which the difference of the labelmaps becomes visually insignificant. 


\section{Results}

We acquired data from 5 patients, who suffer from Hepatocellular carcinoma (HCC) and were treated with TACE. Fig. 2] shows three data sets, the upper row illustrates the original DSA, the lower row overlays with the registered 3D vasculature. The results show that our approach can cope with both variations in 2D as well as in the $3 \mathrm{D}$ data set.

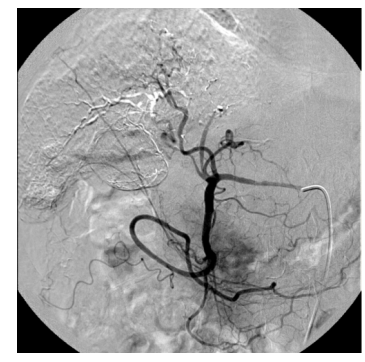

(a)

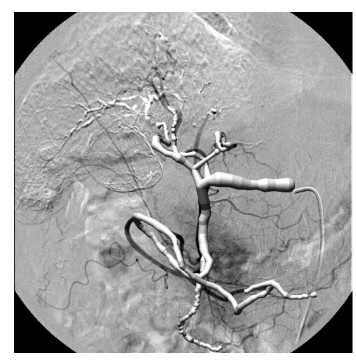

(d)

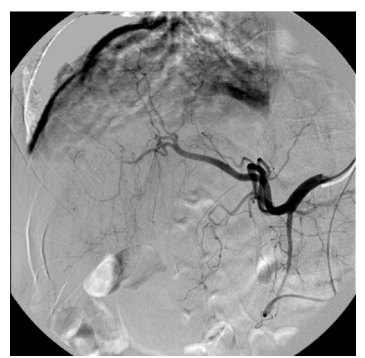

(b)

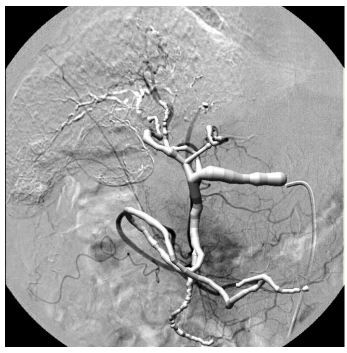

(e)

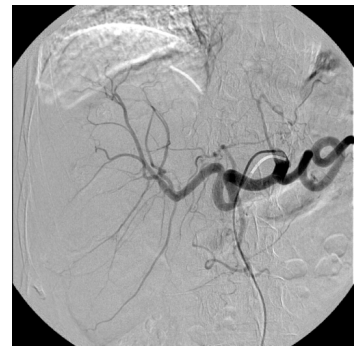

(c)

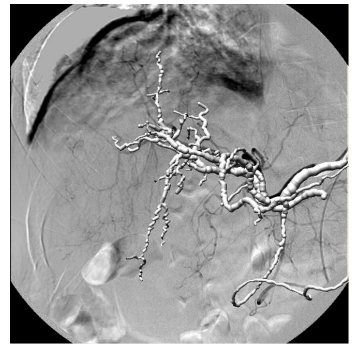

(f)

Fig. 2. Registration results on 3 data sets. 2D vessel trees (upper row) are different as are 3D vasculatures (laid over DSA, lower row). Fig.2(e) shows 3D vasculature with vessels that are not visible in 2D. Large deformations between 2D and 3D can be seen in the lower part of Fig. 2(d) or $2(\mathrm{e})$

For testing accuracy and robustness, reference registration parameters as well as a segmentation of the DSA have been manually created by experienced radiologists for each data set. We added random errors of up to $\pm 5 \mathrm{~mm}$ and $\pm 5^{\circ}$ to each of the translation and rotation parameters, respectively, and invoked the registration method 200 times. The pose parameters are given relative to the origin of the object coordinate system, which was laid in the middle of the moving 3D model. Moreover, as a comparison, we implemented the methods of Jomier et al. [2] and Groher et al. [3] and compared standard deviation $(\sigma)$ and root mean square errors $(\varepsilon)$ for all parameters. 
Table 1. Results of standard deviations $\sigma$ and RMS errors $\varepsilon$ of translations (in $\mathrm{mm}$ ) and rotations (in deg). Deviation and error in $z$-translation are not as significant as those in-plane, or in rotations.

\begin{tabular}{|l|r||lll|lll||lll|lll|}
\hline$\#$ & & $\sigma_{t x}$ & $\sigma_{t y}$ & $\sigma_{t_{z}}$ & $\sigma_{\alpha}$ & $\sigma_{\beta}$ & $\sigma_{\gamma}$ & $\varepsilon_{t_{x}}$ & $\varepsilon_{t y}$ & $\varepsilon_{t_{z}}$ & $\varepsilon_{\alpha}$ & $\varepsilon_{\beta}$ & $\varepsilon_{\gamma}$ \\
\hline \hline 1 & Jomier & 0.76 & 1.80 & 4.66 & 3.48 & 3.17 & 1.60 & 0.78 & 2.21 & $\mathbf{5 . 3 0}$ & 4.75 & 3.60 & 1.66 \\
& Groher & $\mathbf{0 . 2 0}$ & $\mathbf{0 . 0 6}$ & $\mathbf{3 . 4 3}$ & $\mathbf{0 . 0 8}$ & 1.89 & $\mathbf{0 . 1 1}$ & $\mathbf{0 . 3 4}$ & $\mathbf{0 . 2 3}$ & 6.58 & $\mathbf{0 . 2 2}$ & 4.41 & $\mathbf{0 . 3 8}$ \\
& EBM & 0.54 & 2.68 & 34.63 & 3.79 & $\mathbf{1 . 8 8}$ & 1.25 & 0.54 & 2.70 & 36.12 & 3.79 & $\mathbf{1 . 9 7}$ & 1.25 \\
\hline 2 & Jomier & 0.74 & 0.93 & 5.06 & 4.84 & 3.18 & 0.58 & 0.78 & 3.46 & 5.38 & 8.33 & 3.18 & 0.78 \\
& Groher & 0.16 & 1.33 & 4.52 & 4.80 & 2.59 & 1.27 & 1.24 & 1.49 & 28.48 & 5.28 & 3.85 & 2.33 \\
& EBM & $\mathbf{0 . 0 4}$ & $\mathbf{0 . 1 6}$ & $\mathbf{1 . 9 9}$ & $\mathbf{0 . 4 5}$ & $\mathbf{0 . 1 8}$ & $\mathbf{0 . 0 4}$ & $\mathbf{0 . 0 7}$ & $\mathbf{0 . 2 5}$ & $\mathbf{3 . 7 3}$ & $\mathbf{0 . 7 2}$ & $\mathbf{0 . 3 5}$ & $\mathbf{0 . 0 4}$ \\
\hline 3 & Jomier & 3.34 & 2.03 & $\mathbf{7 . 3 9}$ & 4.43 & 4.37 & $\mathbf{2 . 0 4}$ & 4.68 & 2.96 & $\mathbf{7 . 4 3}$ & 5.62 & 4.39 & $\mathbf{2 . 0 7}$ \\
& Groher & $\mathbf{2 . 3 2}$ & 4.56 & 43.72 & $\mathbf{3 . 8 4}$ & 3.98 & 4.78 & 6.72 & 6.21 & 62.07 & $\mathbf{5 . 1 9}$ & 11.58 & 4.87 \\
& EBM & 3.55 & $\mathbf{1 . 5 3}$ & 24.11 & 7.47 & $\mathbf{3 . 3 3}$ & 4.62 & $\mathbf{3 . 5 4}$ & $\mathbf{1 . 5 4}$ & 24.36 & 7.49 & $\mathbf{3 . 3 3}$ & 4.62 \\
\hline 4 & Jomier & 22.45 & 6.65 & 13.73 & 7.74 & 24.64 & 9.15 & 23.18 & 6.84 & 13.81 & 8.40 & 25.41 & 9.16 \\
& Groher & 6.30 & 0.89 & 55.10 & 4.55 & 6.82 & 4.09 & 9.05 & 2.02 & 70.69 & 7.54 & 14.73 & 4.99 \\
& EBM & $\mathbf{0 . 9 5}$ & $\mathbf{0 . 1 8}$ & $\mathbf{9 . 4 1}$ & $\mathbf{0 . 2 9}$ & $\mathbf{1 . 9 6}$ & $\mathbf{0 . 1 0}$ & $\mathbf{1 . 0 9}$ & $\mathbf{0 . 1 9}$ & $\mathbf{1 3 . 0 4}$ & $\mathbf{0 . 3 6}$ & $\mathbf{2 . 6 2}$ & $\mathbf{0 . 1 0}$ \\
\hline $\mathbf{5}$ & Jomier & 26.35 & 16.37 & $\mathbf{1 0 . 3 4}$ & 7.15 & 13.60 & 5.81 & 26.66 & 16.37 & $\mathbf{1 0 . 8 7}$ & 7.25 & 18.63 & 6.07 \\
& Groher & 20.65 & 1.82 & 148.41 & 14.88 & 12.29 & 15.10 & 52.74 & 3.39 & 422.62 & 28.76 & 16.95 & 24.40 \\
& EBM & $\mathbf{1 . 2 2}$ & $\mathbf{0 . 7 4}$ & 16.83 & $\mathbf{3 . 0 5}$ & $\mathbf{2 . 0 7}$ & $\mathbf{0 . 6 7}$ & $\mathbf{1 . 4 1}$ & $\mathbf{0 . 7 4}$ & 20.19 & $\mathbf{3 . 0 5}$ & $\mathbf{2 . 4 4}$ & $\mathbf{0 . 7 6}$ \\
\hline \hline \multirow{2}{*}{ Avrg. } & Jomier & 10.73 & 5.60 & $\mathbf{8 . 2 3}$ & 5.53 & 9.79 & 3.83 & 11.21 & 6.37 & $\mathbf{8 . 5 6}$ & 6.87 & 11.04 & 3.95 \\
& Groher & 5.93 & 1.70 & 51.04 & 5.63 & 5.5 & 5.07 & 14.02 & 2.67 & 118.09 & 9.39 & 10.30 & 7.39 \\
& EBM & $\mathbf{1 . 2 6}$ & $\mathbf{1 . 0 6}$ & 17.39 & $\mathbf{3 . 0 1}$ & $\mathbf{1 . 8 8}$ & $\mathbf{1 . 3 4}$ & $\mathbf{1 . 3 3}$ & $\mathbf{1 . 0 8}$ & 19.49 & $\mathbf{3 . 0 8}$ & $\mathbf{2 . 1 4}$ & $\mathbf{1 . 3 5}$ \\
\hline
\end{tabular}

For the method of Groher et al. we used the reference 2D segmentation for 2D graph creation and registration. The result of this study is summarized in table 1 . It can be seen that error and deviation in z-translation is sometimes outperformed by the other two methods, however, the more important in-plane translation and rotations have less error in our method. The large errors of Jomier's and Groher's method in the last two data sets can be explained with a "subset" property. In the first three data sets the 3D vasculature is a "subset" of the $2 \mathrm{D}$ vasculature, whereas in the last two data sets this property is not fulfilled, i.e. the 3D vasculature shows branches that are not visible in $2 \mathrm{D}$. The results show that our method is more robust with respect to variability in both dimensions. The number of iterations of our algorithm usually lies between 2 and 5 . The runtime (analyzed on a Intel Core2Duo 2.6 GHz machine) splits into (bothat-) filtering $(28.5 \mathrm{sec})$, seed point extraction $(1.0 \mathrm{sec})$, region growing $(0.3 \mathrm{sec})$, centerline extraction $(2.7 \mathrm{sec})$, exhaustive initialization $(34.9 \mathrm{sec})$, iteration $3(12.9 \mathrm{sec})$, where all runtimes have been averaged over the 5 patient data sets and the iteration runtime over the number of iterations. Altogether, applying the registration takes $1.5-2 \mathrm{~min}$. The two critical stages are filtering and exhaustive initialization - both can be further optimized numerically.

\section{Conclusion}

We have developed a method for 2D-3D registration of angiographic data. Our emphasis lies on a fully automatic registration once the interventionalist starts the treatment. We believe that a 2D segmentation can yield a more robust (feature-based) registration with high capture range. Motivated by an ML formulation of a combined segmentation/registration, we derived a generic method for estimating the $2 \mathrm{D}$ labelmap and the

\footnotetext{
${ }^{3}$ Region growing, centerline extraction, and pose optimization.
} 
registration parameters iteratively linked by a common probability map. In this probabilistic framework, we keep the freedom to choose any segmentation or registration technique. Unlike other approaches, we keep user interaction low while high capture range and robustness against vessel variability and deformation are maintained. With the segmentation-driven registration, we create a common feature space and thus oneto-one correspondence of vessel features. Hence, we can visualize catheter locations and roadmaps in 3D and 2D simultaneously (see supplementary material). Application to different medical procedures and tests of alternative 2D segmentation and/or registration steps will follow the work in the future. With these future tests, we will also assess the impact of all processing steps, which are performed in the iteration.

Acknowledgements. This research was funded by an academic grant from Siemens Medical Solutions Angiography/X-Ray division, Forchheim, Germany. The authors would like to thank in particular K. Klingenbeck-Regn and M. Pfister for their continuous support.

\section{References}

1. Turgeon, G.A., Lehmann, G., Guiraudon, G., Drangova, M., Holdsworth, D., Peters, T.: 2D$3 \mathrm{D}$ registration of coronary angiograms for cardiac procedure planning and guidance. Medical Physics 32, 3737-3749 (2005)

2. Jomier, J., Bullitt, E., van Horn, M., Pathak, C., Aylward, S.: 3D/2D model-to-image registration applied to tips surgery. In: Larsen, R., Nielsen, M., Sporring, J. (eds.) MICCAI 2006. LNCS, vol. 4190, pp. 662-670. Springer, Heidelberg (2006)

3. Groher, M., Padoy, N., Jakobs, T., Navab, N.: New CTA protocol and 2D-3D registration method for liver catheterization. In: Larsen, R., Nielsen, M., Sporring, J. (eds.) MICCAI 2006. LNCS, vol. 4190, pp. 873-882. Springer, Heidelberg (2006)

4. Hamadeh, A., Lavalle, S., Cinquin, P.: Automated 3-dimensional computed tomographic and fluoroscopic image registration. Computer Aided Surgery 3, 11-19 (1998)

5. Bansal, R., Staib, L.H., Chen, Z., Rangarajan, A., Knisely, J., Nath, R., Duncan, J.S.: Entropybased, multiple-portal-to-3DCT registration for prostate radiotherapy using iteratively estimated segmentation. In: Taylor, C., Colchester, A. (eds.) MICCAI 1999. LNCS, vol. 1679, pp. 567-578. Springer, Heidelberg (1999)

6. Brox, T., Rosenhahn, B., Weickert, J.: Three-dimensional shape knowledge for joint image segmentation and pose estimation. In: Kropatsch, W.G., Sablatnig, R., Hanbury, A. (eds.) Pattern Recognition. LNCS, vol. 3663, pp. 109-116. Springer, Heidelberg (2005)

7. Pohl, K.M., Fisher, J., Grimson, Kikinis, Ron, Wells, W.M.: A bayesian model for joint segmentation and registration. NeuroImage 31, 228-239 (2006)

8. Dempster, A.P., Laird, N.M., Rubin, D.B.: Maximum likelihood from incomplete data via the EM algorithm. J. of the Royal Statistical Soc. Series B 39, 1-38 (1977)

9. Can, A., Shen, H., Turner, J., Tanenbaum, H., Raysam, B.: Rapid automated tracing and feature extraction from retinal fundus images using direct exploratory algorithms. IEEE Transactions on Information Technology in Biomedicine 3, 125-138 (1999) 